\title{
Electrical stimulation enhances reinnervation after nerve injury
}

\author{
Michael P Willand \\ Department of Surgery, Division of Plastic Reconstructive Surgery. The Hospital for Sick \\ Children, Toronto, Canada
}

\begin{abstract}
Electrical muscle stimulation following peripheral nerve injury has been a controversial method of treatment due primarily to the inconsistent literature surrounding it. In this presentation transcript I outline ongoing experiments investigating a clinically translatable daily muscle stimulation paradigm in rats following nerve injury. Results show that reinnervation of muscle and functional behavioural metrics are enhanced with daily stimulation with upregulation of intramuscular neurotrophic factors as a potential mechanism. In addition, the impact of stimulation on terminal sprouting, a mentioned negative aspect of electrical muscle stimulation, was a minor contributor to long term functional reinnervation of stimulated muscles in our studies.

Key Words: Electrical muscle stimulation, nerve injury, axon sprouting, motor unit number estimation, EMG, intramuscular neurotrophic factors
\end{abstract}

Eur J Transl Myol - Basic Appl Myol 2015; 25 (4): 243-248

My typescript will be a little different from those of the present EJTM Special "Mobility in Elderly". It focuses, indeed, on nerve injuries and reinnervation and it is not a human study but on rats. I will not go into details too much because I have a lot of information. Briefly, there are a few negative papers that say electrical stimulation (ES) results in poor or abnormal reinnervation. In more than 100 papers on the topic, two only are negative, but people tend to focus on those, in particular on the one that refers the results of "chronic stimulation", that is of 12 to 24 hours per day, which is a lot. A 1978 paper that always comes up reports effects of 10-12 hours per day of ES. ${ }^{1}$ The major finding in this paper is reduction in terminal sprouting. So, what is terminal sprouting? The authors severed part of a nerve and stimulated the remaining: just one axon or several axons were removed in this partially innervated muscle. Authors found that the intact neuromuscular junctions create terminal sprouts, while electrical stimulation reduces the sprouting. ${ }^{1}$ In 2003 another group investigated 24 hours per day of stimulation following partial or compete denervation. They wanted to find out if terminal sprouting was being inhibited. In this case they found that terminal Schwann cells sit on top of the neuromuscular junctions. Usually, in a partial nerve injury, the terminal Schwann cell sprouts bridges, and this bridge acts as a pathway for the terminal axon sprouting. Stimulation reduced this bridging. ${ }^{2}$ In a 2009 paper, the facial nerve was transected and repaired in rats and electrostimulated with three 5 minutes sessions per week. The current amplitude was so low that muscle contractions were not elicited. Surprisingly, they found out that there was a reduction of reinnervation. ${ }^{3}$ There is also a paper with 20 contractions every 48 hours almost nothing. They also found out that the stimulated group was worse. ${ }^{4}$

These are some of the studies suggesting that electrical stimulation is bad - "don't do it".

\section{A moderate ES paradigm}

What I have been working on is something in between. The sessions take place 5 days per week with a duration of 1 hour delivering 600 contractions. We stimulate the gastrocnemius muscle at $100 \mathrm{~Hz}$, an essentially fast twitch muscle. This is how we deliver the paradigm: basically, the rats are restrained in a plastic tube, in which they are free to move. We exposed the right leg which is the experimental leg. What we do is restrain five rats at the same time. You can actually see what these contractions here look like - once every 6 seconds. So, here is what we did to test this paradigm. What happens if we use this paradigm after nerve transection and stimulate the muscle for two weeks? For this we had two groups of rats undergoing nerve transection and immediate repair. One group had the right hind limb stimulated for two weeks starting 2 days after surgery.

\section{Assessing early reinnervation following ES}

We made two incisions: One for nerve transection and one to implant the electrodes. The two parameters we 


\section{MUNE (2 Weeks ES)}

- The number of motor units were estimated using EMG methods. Stimulated muscles showed a more than 2-fold increase in motor unit numbers.
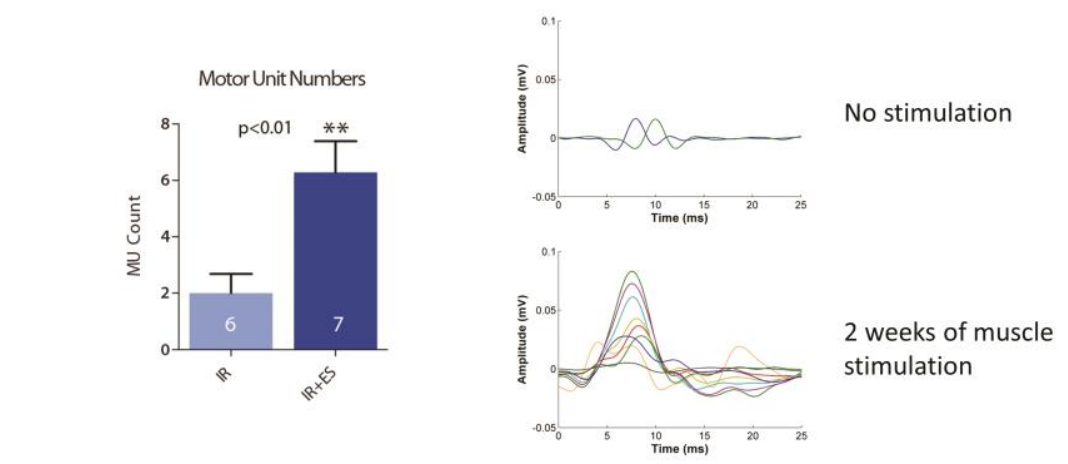

Fig 1. MUNE was performed using EMG on rat gastrocnemius muscles 2 weeks following tibial nerve transection and immediate repair (IR) or transection and immediate repair with daily electrical muscle stimulation $(I R+E S)$. Motor unit $(M U)$ counts were significantly higher in stimulated muscles. EMG responses were robust in stimulated muscle in comparison to non-stimulated muscle.

looked for were motor unit number estimation (MUNE) and immunohistochemistry for endplate staining. For MUNE there are two ways to estimate it: by electromyography (EMG) or by muscle force. We preferred EMG. What we did was to connect the stimulator proximal to the injury and then two electrodes in the muscle. What you get are templates. We collected between 15 to 20 templates. Then we can calculate the MU count by dividing the maximum response by the average response from the 15-20 templates. After two weeks the motor unit numbers in the muscle were significantly higher, a more than 2-fold increase (Figure 1). When you look at the EMG you can see that there is a more robust EMG. ${ }^{3-5}$ Clearly, there is more reinnervation taking place. By immunohistochemistry, the endplates may be stained in red, while in green may be labelled the axons. It is pretty clear that the stimulated muscles contained greater numbers of innervated endplates and intramuscular axons. The last thing we did was to look at longitudinal sections. They showed a greater number of axons in the distal stump of stimulated muscle. Observing a nerve section from a rat that did not receive ES, proximal to be nerve injury all the axon appeared intact while at the injury site, even at high resolution, the site is pretty dark, which means there were not many axons growing. In contrast, in a stimulated rat at the site of injury there were many more axons at both high and low resolution. It was pretty clear that our muscle stimulation paradigm of early stimulation works and increases motor reinnervation. A further question is: what happens in the long run if we keep stimulating throughout the reinnervation period?

\section{Assessing long-term reinnervation and functional recovery}

The answer is a next study, in which ES lasted for 1, 2 or 3 months. Everything was the same but this time we have quantified the reinnervation. We also looked at functional metrics with the walking track analysis and tapered beam tests. The results for MUNE were really nice. The separation extends from 1 to 3 months (Figure 2). We have a significant increase in $\mathrm{MU}$ numbers. In the end it is not different from a normal muscle. $^{6}$

Let's go a little bit more into immunohistochemistry. We analyzed a minimum of 300 endplates per muscle. We divided them into 5 categories: normal reinnervation, reinnervated by a nodal sprout, reinnervated by a terminal sprout, reinnervated but unknown (we don't know where the axon was from) and of course there were denervated endplates. The results show that the majority of the endplates were reinnervated. In fact, if you combine all those reinnervated only $10 \%$ of the muscle endplates were denervated, the rest were reinnervated. The sprouting events were seldom. So, it might be that electrical stimulation reduces terminal sprouting, we could say this is bad, but look at the percentage: it's only 5\% (Figure 3). 


\section{MUNE $(1,2$, or 3 Months ES)}

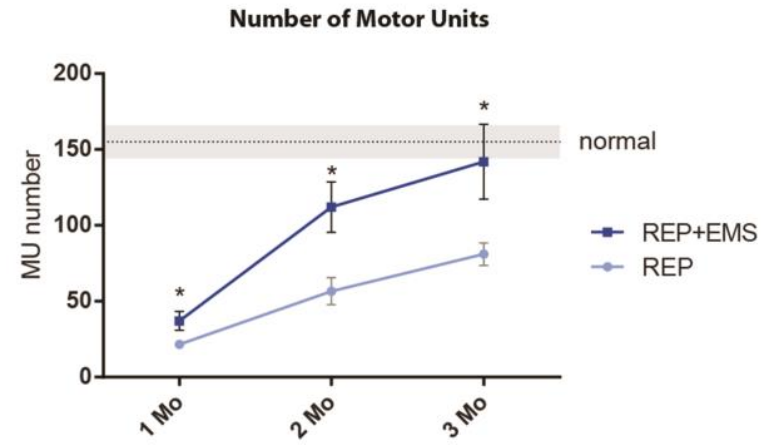

Fig 2. MUNE was performed using EMG on rat gastrocnemius muscles 1, 2, or 3 months following tibial nerve transection and immediate repair (REP) or transection and immediate repair with daily electrical muscle stimulation $(R E P+E M S)$. Motor unit $(M U)$ counts were significantly higher at every time point. At 3 months post nerve repair stimulated muscles has motor unit numbers no different than uninjured controls.

The next thing we analyzed was the functional metrics over 3 months. Every week, for three months we took behavioral tests to observe functional recovery. The walking track analysis was for measuring overground locomotion and the tapered beam test for measuring skilled locomotion. For the walking track test we dip

\section{Reinnervation Summary (3 Months of ES)}

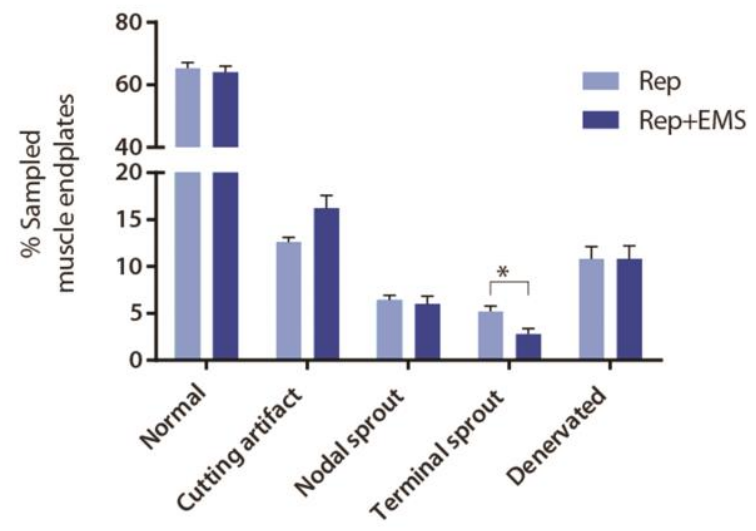

Fig 3. Gastrocnemius muscle endplates were examined for reinnervation 3 months following tibial nerve transection and repair with $(R E P+E M S)$ or without $(R E P)$ electrical muscle stimulation. A minimum of 300 endplates per muscle were examined. The vast majority (approximately 90\%) of endplates were reinnervated with $60 \%$ having one axon per endplate coming from a nerve trunk (normal reinnervation). Sprouting accounted for a very small proportion of reinnervation (approximately 10\%). Terminal sprouting was significantly reduced in stimulated muscle (2.5\% of sampled endplates) in comparison to non-stimulated (5\% of sampled endplates). 


\section{Tapered Beam Test (3 Months of ES)}

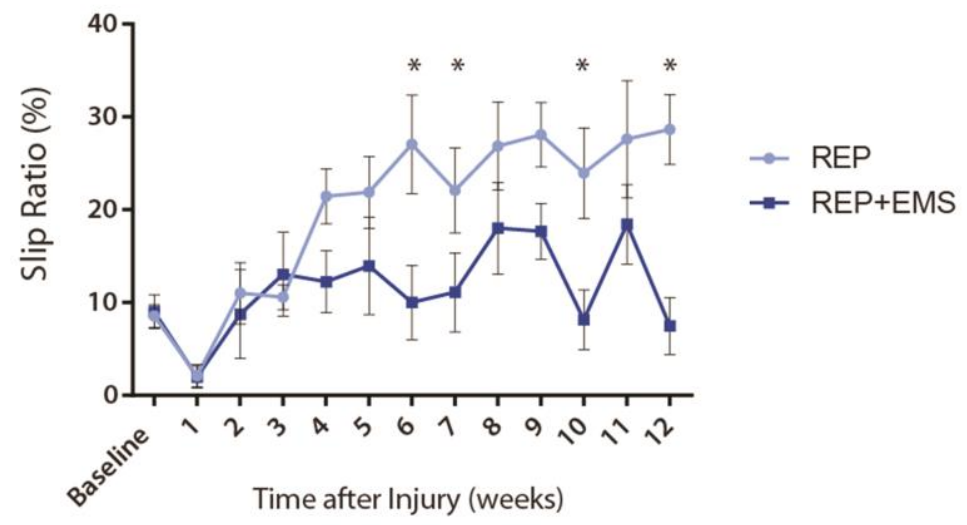

Willand et al. Neurorehabilitation and Neural Repair. 2015 - in press.

Fig 4. Rats were assessed weekly over a 3 month period following tibial nerve transection and immediate repair using a tapered beam test. Foot slips were quantified and expressed as a percentage of the number of steps taken to reach the end of the beam. Rats receiving daily gastrocnemius electrical stimulation (REP+EMS) had a significantly lower slip ratio in comparison to rats in the non-stimulated group (REP).

the rat's feet in ink and let them walk across a piece of paper. What we had to measure were the toe spread, the print length and the intermediate toe spread and you plug these data into a formula and you get a number called the tibial function index. We didn't have much separation between the groups.

We found out that it is not a really good metric for tibial nerve injuries. The next test we did was the tapered beam test. Basically, this beam is used to assess skilled locomotion and sensorimotor deficits. The rats have to walk on it. We take notes on the foot-faults (slips). We have full slips and half-slips, which allows us to calculate the slip percentage. After surgery the rats were not walking properly. After 6 weeks we noticed the rats stopped using their backwards sliding motion they used before. Finally, a video at 3 months showed that the unstimulated rats are still slipping a lot. The stimulated rats are not slipping as much as the others. After 4 weeks we have a separation. The stimulated rats have much better recovery and are considerably better. Week one is the worst for both groups (Figure 4).

After 3-4 weeks they start going back to normal. At week 5 they are using the sliding motion anymore. After week 4 there is a separation taking place between unstimulated and stimulated groups of rats.

In summary it is clear proof that stimulation increases functional recovery and that he reinnervation is not adversely affected at the neuromuscular junction level.

\section{Intramuscular neurotrophic factors}

What is the reason for the improved results in stimulated rats? The early enhancement of reinnervation can be potentially explained by evidence that stimulation of the proximal nerve stump following injury can increase the rate of axonal outgrowth across the injury site. How can this make sense in our model? We started stimulation two days after surgery. This allows time for degeneration to take place. Could the trophic factors be a reason for the improved reinnervation? The Schwann cells may be responsive to electrical stimulation and upregulate trophic factors. But there is no real evidence, yet. Data from earlier this week on BDNF and GDNF show that both were significantly upregulated 2 weeks after injury (Figure 5 ). The stimulated group had greater amounts of BDNF and GDNF.

In the past we looked at 3 months of denervation. No nerve was there. We did the same with electrical stimulation. BDNF and GDNF were higher and also NGF was significantly different. This is something that needs to be looked at in the future.

Further things we want to look at are quite interesting. We want to know if other paradigms can produce similar results. If we change the muscle, intensity and so on, can we achieve similar results? Also, we want to examine the distal stump factors during the first weeks of stimulation following nerve injury and repair. The 


\section{Trophic Factors (2 weeks of ES)}
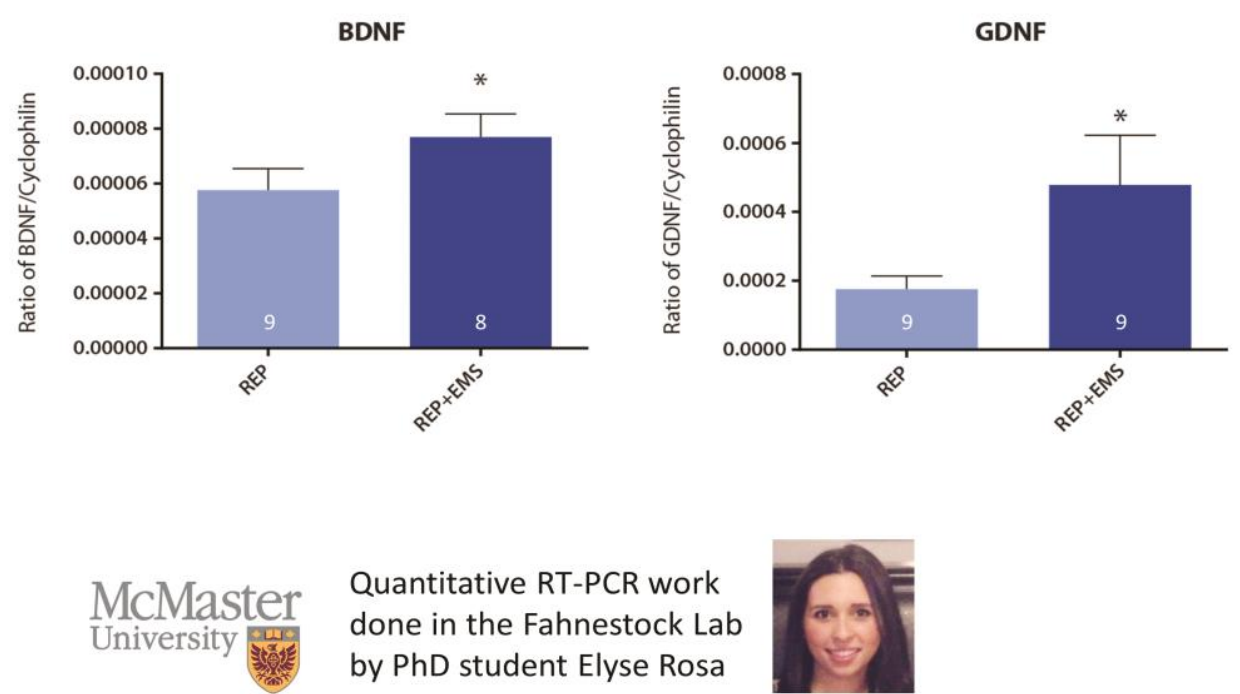

Quantitative RT-PCR work done in the Fahnestock Lab by PhD student Elyse Rosa

Fig 5. Quantitative RT-PCR was performed on gastrocnemius muscle samples taken from rats that were stimulated daily for 2 weeks $(R E P+E M S)$ or not (REP) following tibial nerve transection and repair. Levels of brain-derived neurotrophic factor (BDNF) and glial-derived neurotrophic factor (GDNF) mRNA were normalized to cyclophilin. Daily electrical muscle stimulation significantly upregulated both GDNF and BDNF mRNA levels after only 2 weeks of stimulation.

next point has never been tried before. What about the combination of proximal nerve stimulation during nerve repair with daily muscle stimulation? Is there a synergistic effect? Will it double up the response?

\section{A rodent model to assess synkinetic reinnervation following daily ES}

Does muscle stimulation affect synkinesis (nerve misdirection)? If you cut a nerve then the axons are not going to grow back to the original muscles. They go back in a random fashion. This is typically evident in the facial nerves. When someone tries to smile their eye closes. That is synkinesis. There is some evidence in the larynx of dogs that intramuscular stimulation using a "natural" pattern can allow for original axons to reconnect. The problem is no one has ever done it this way. This is just a graphic of what we are doing. We have the soleus and the lateral gastrocnemius and they are innervated by a common branch - the lateral gastrocnemius-soleus nerve. We inject some blue dye into the soleus and this goes to the spinal cord after some time. Then we do a nerve transection and repair and connect the stimulator to the soleus. After 2 months we cut that soleus nerve and we basically put the nerve in a well, for which we use red color. Ideally, all the axons labeled by injection will be labeled when you dip this nerve in the well. It is actually not the case. This is an injection with blue dye. The idea behind all this is to take all these spinal cords and we take some sections and we quantify these labeled neurons. The idea it so make 3D models. We want to see where these motoneurons are and also we want to see if they come from the same population after injury. That's where the whole project is now.

\section{Questions from the audience and answers}

Audience question: How stable is the reinnervated phenotype if you stop the electrical stimulation?

Willand: Interestingly, I published a paper on repair last year. There we stopped stimulation. That is, we stimulated for one month and stopped for two months. There was already an improvement and the effect was present. It goes with the timing, right? So if you continue to stimulate and then stop you lose the benefit. We definitely need a better controlled study for that. So far, it looks like there is, but it still needs some more research. 


\section{ES enhances reinnervation after nerve injury}

Eur J Transl Myol - Basic Appl Myol 2015; 25 (4): 243-248

Audience question: We have heavy constraints from our surgeons because they are not open to stimulation after the surgery and during repair. Did you stimulate during the first hours after the surgery?

Willand: We stimulated two days after surgery.

Audience question: Now you moved from immediate repair to two days after repair.

Willand: It was always two days after repair in my rat experiments. Clinically, I can say that we had four pediatric patients that received nerve stimulation. It was no problem. We did the stimulation on these patients during nerve repair for $1 \mathrm{~h}$ and it was no problem. The biggest problem is that in humans everything takes so long. It takes a while to see a benefit. It is possible to do it and it is not difficult. We used an old Grass stimulator and it worked.

Audience question: How long do you have to wait to see improvement?

Willand: That is the big question. We don't know yet.

Audience question: Have it been single twitches for the stimulation of the hind leg?

Willand: These contractions were 400 milliseconds long at $100 \mathrm{~Hz}$.

Audience question: There are no bursts?

Willand: They are fast. So, it is a fast muscle contraction. Kern: We have to take in account, if we stimulate seniors, we should avoid any negative effect on the regeneration process. Therefore, we invited Mike Willand. For me it is important that we avoid damage and that we do not influence the nerve regeneration in seniors. So, I am very thankful and happy. When I saw this for the first time, it was not clear if the muscle stimulation was enhancing or inhibiting the axon regeneration. Here we see that stimulation after nerve injury is in fact enhancing the recovery. Now we can be sure that the FES stimulation will not disturb the eventual nerve reinnervation process.

\section{Acknowledgement}

The work here outlined was funded by MED-EL GmbH.

\section{Corresponding Author}

Michael P Willand, The Hospital for Sick Children, Toronto, Ontario, Canada

E-mail: mike.willand@gmail.com

\section{References}

1. Ironton R, Brown MC, Holland RL. Stimuli to intramuscular nerve growth. Brain Res. 1978 Nov 10;156(2):351-4.

2. Love FM, Son Y-J, Thompson WJ. Activity alters muscle reinnervation and terminal sprouting by reducing the number of Schwann cell pathways that grow to link synaptic sites. J Neurobiol. 2003 Mar;54(4):566-76.

3. Sinis N, Horn F, Genchev B, et al. Electrical stimulation of paralyzed vibrissal muscles reduces endplate reinnervation and does not promote motor recovery after facial nerve repair in rats. Ann Anat. 2009;191:356-70.

4. Gigo-Benato D, Russo TL, Geuna S, et al. Electrical stimulation impairs early functional recovery and accentuates skeletal muscle atrophy after sciatic nerve crush injury in rats. Muscle Nerve 2010;41:685-693.

5. Willand MP, Zhang JJ, Chiang CD, BorschelGH, Gordon T. Electrical muscle stimulation increases early reinnervation following nerve injury and immediate repair. 2013 6th Int IEEEEMBS Conf Neural Eng NER. 2013 Nov;315-8.

6. Willand MP, Chiang CD, Zhang JJ, Kemp SWP, Borschel GH, Gordon T. Daily Electrical Muscle Stimulation Enhances Functional Recovery Following Nerve Transection and Repair in Rats. Neurorehabil Neural Repair. 2014 Dec $11 ; 1545968314562117$. 